\title{
Targeting the NCOA3-SP1-TERT axis for tumor growth in hepatocellular carcinoma
}

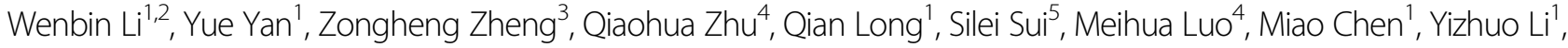 \\ Yijun Hua', Wuguo Deng ${ }^{1}$, Renchun Lai ${ }^{1}$ and Liren Li ${ }^{1}$
}

\begin{abstract}
Hepatocellular carcinoma (HCC) has a high mortality rate and lacks an effective therapeutic target. Elevated expression of human telomerase reverse transcriptase (TERT) is an important hallmark in cancers, but the mechanism by which TERT is activated differentially in cancers is poorly understood. Here, we have identified nuclear receptor coactivator-3 (NCOA3) as a new modulator of TERT expression and tumor growth in HCC. NACO3 specifically binds to the TERT promoter at the -234 to -144 region and transcriptionally activates TERT expression. NCOA3 promotes HCC cell growth and tumor progression in vitro and in vivo through upregulating the TERT signaling. Knockdown of NACO3 suppresses HCC cell viability and colony formation, whereas TERT overexpression rescues this suppression. NCOA3 interacts with and recruits SP1 binding on the TERT promoter. Knockdown of NCOA3 also inhibits the expression of the Wnt signaling-related genes but has no effect on the Notch signaling-targeting genes. Moreover, NCOA3 is positively correlated with TERT expression in HCC tumor tissues, and high expression of both NCOA3 and TERT predicts a poor prognosis in HCC patients. Our findings indicate that targeting the NCOA3-SP1-TERT signaling axis may benefit HCC patients.
\end{abstract}

\section{Introduction}

Hepatocellular carcinoma (HCC) is one of the most common cancers and a leading cause of cancer death worldwide ${ }^{1}$. The rising incidence and mortality rate of HCC in most countries are due to an increased rate of chronic Hepatitis $\mathrm{C}$ virus infection, alcohol abuse, and obesity $^{2,3}$. Although therapeutic approaches for HCC have been improved in recent years, the long-term prognosis of patients with $\mathrm{HCC}$ is still poor ${ }^{4}$. The molecular mechanisms leading to the development of HCC are

\footnotetext{
Correspondence: Wuguo Deng (dengwg@sysucc.org.cn) or Renchun Lai (lairch@sysucc.org.cn) or Liren Li (lilr@sysucc.org.cn)

${ }^{1}$ Sun Yat-sen University Cancer Center; State Key Laboratory of Oncology in South China; Collaborative Innovation Center of Cancer Medicine, Guangzhou, China

${ }^{2}$ Institute of Neuroscience and Department of Neurology of the Second Affiliated Hospital of Guangzhou Medical University, Key Laboratory of Neurogenetics and Channelopathies of Guangdong Province and the Ministry of Education of China, Guangzhou, China

Full list of author information is available at the end of the article

These authors contributed equally: Wenbin Li, Yue Yan, Zongheng Zheng

Edited by $\mathrm{G}$. Ciliberto
}

complicated, consist of various genetic and epigenetic changes, and are associated with altered signaling pathways $^{5}$. Among these molecular events, elevated expression of human telomerase reverse transcriptase (TERT) is an important alteration in HCC and a hallmark of human cancer $^{6,7}$.

TERT is the catalytic component of telomerase and extends telomeres with its RNA partner (telomerase RNA component). TERT can directly regulate the various hallmarks of cancer, which include sustaining proliferative signaling, evading growth suppressors, inducing angiogenesis, resisting cell death, activating invasion and metastasis, tumor-promoting inflammation, and enabling replicative immortality ${ }^{8}$. What's more, TERT also acts as a regulator of the transcription of genes involved in cancer cell growth and proliferation, independently of its role in telomeres ${ }^{9}$. TERT is highly transcribed in germ cells, stem cells, and cancer cells, but is barely detectable in most somatic cells ${ }^{10}$. In cancer cells, TERT is transcriptionally reactivated by oncogenic transcription factors such as

\section{(c) The Author(s) 2020}

(c) (i) Open Access This article is licensed under a Creative Commons Attribution 4.0 International License, which permits use, sharing, adaptation, distribution and reproduction in any medium or format, as long as you give appropriate credit to the original author(s) and the source, provide a link to the Creative Commons license, and indicate if changes were made. The images or other third party material in this article are included in the article's Creative Commons license, unless indicated otherwise in a credit line to the material. If material is not included in the article's Creative Commons license and your intended use is not permitted by statutory regulation or exceeds the permitted use, you will need to obtain permission directly from the copyright holder. To view a copy of this license, visit http://creativecommons.org/licenses/by/4.0/. 
Myc, NF- $\mathrm{BB}, \quad \beta$-catenin, and $\mathrm{SP} 1^{8,11,12}$, and TERT expression is elevated in HCC patients and positively correlated with the incidence and development of $\mathrm{HCC}^{13,14}$. However, the detailed molecular mechanism of TERT transcription activation in HCC is not very clear. A key question is whether specific oncogenic transcription factors or coactivators activate the transcription of TERT in $\mathrm{HCC}$ to promote HCC development.

Nuclear receptor coactivators-3 (NCOA3, also known as SRC-3 or AIBI) is a member of the steroid receptor coactivator (SRC)/p160 gene family, and it was identified from an amplified region on the long arm of chromosome 20 (20q) in breast cancer tissue ${ }^{15}$. NCOA3 protein has multiple structural and functional domains, including an N-terminal basic helix-loop-helix-Per/ARNT/Sim (bHLH-PAS) domain, a nuclear receptor-interacting domain, and a C-terminal transcriptional activation domain (AD) with histone acetyltransferase (HAT) and p300/CBP binding activities ${ }^{16}$. The SRC/p160 gene family has three homologous members, NCOA1/SRC-1, NCOA2/SRC-2, and NCOA3/SCR- $3^{17}$. Unlike NCOA1 and NCOA2, NCOA3 mediates not only the transcriptional activity of nuclear receptors, but also other transcription factors, such as SP1, c-Myc, NF-kB, HIF1 $\alpha$, CREB, AP-1, E2F1, and PEA3 ${ }^{18-22}$.

SP1, a key transcription factor of TERT, can bind to the GC-box of the TERT promoter and cooperate with c-Myc to active TERT transcription. Some regulators, including tumor activators and suppressors, can regulate SP1 binding activity to TERT promoter to enhance or inhibit TERT transcription in $\mathrm{HCCs}^{23-26}$.

NCOA3 is highly expressed in many human cancers, including breast, prostate, lung, pancreas, and colorectal carcinoma $^{27-29}$. Overexpression of NCOA3 has been reported to enhance cell proliferation and invasion to promote HCC progression ${ }^{30}$, but the exact molecular mechanism of NCOA3-regulated HCC growth remains elusive.

In this study, we discovered and identified a new mechanism involved hTERT regulation and HCC growth. We designed a $5^{\prime}$ biotin-labeled TERT promoter ( -387 to -137) probe to pull down the TERT promoter binding proteins by biotin-streptavidin-beads pull-down (BSBP) method and identified NCOA3 as a novel TERT promoter binding protein binds to the -234 to -144 region of the TERT promoter in HCC cells. We demonstrated that NCOA3 enhanced TERT expression in HCC cells, and NCOA3/TERT signaling promoted HCC cell growth in vitro and in vivo. We further revealed that NCOA3 interacted with SP1 to regulate TERT expression and HCC cell growth. Finally, our study showed that both NCOA3 and TERT were highly expressed in HCC patients and associated with poor clinical outcomes. Thus, our results uncovered a novel mechanism of HCC growth regulation by activating the NCOA3/SP1/hTERT axis, and indicating that the NCOA3-Sp1-TERT axis could be a potential therapeutic target for $\mathrm{HCC}$.

\section{Materials and methods \\ Cell lines and cell culture}

HCC cells (Hep3B, HepG2, BEL-7402, and SNU-449) were obtained from the American Type Culture Collection (ATCC, Manassas, VA), human immortalized hepatic epithelial cell lines LO2 was obtained from Sun Yat-Sen University Cancer Center. Cells were cultured in Dulbecco's Modified Eagle Medium (Invitrogen, Carlsbad, CA) or RPMI-1640 (Gibco BRL, Grand Island, NY) supplemented with $10 \%$ fetal bovine serum (Invitrogen), $100 \mu \mathrm{g} / \mathrm{ml}$ penicillin, and $100 \mathrm{U} / \mathrm{ml}$ streptomycins. All cells were maintained in a humidified atmosphere and $5 \%$ $\mathrm{CO}_{2}$ at $37^{\circ} \mathrm{C}$.

\section{Antibodies and reagents}

Anti-NCOA3 antibody (\#2126), anti-SP1 (D4C3) antibody (\#9389), and anti-Acetylated-Lysine antibody (\#9441) were purchased from Cell Signaling Technology (Danvers, MA). Anti-TERT antibody (\#NB110-89471) was from Novus Biologicals (Littleton, CO). Anti-NCOA3 ChIP grade antibody (\#ab2782) was from Abcam (Cambridge, MA). Anti-GAPDH antibody (\#10494-1-AP), antihistone H3 antibody (\#17168-1-AP), horseradish peroxidase (HRP)-conjugated Goat Anti-Rabbit IgG antibody (\#SA00001-2), Alexa Fluor 488 conjugated Goat AntiMouse IgG antibody (\#SA00006-1), Alexa Fluor 594 conjugated Goat Anti-Rabbit IgG antibody (\#SA00006-4) were from Proteintech (Wuhan, China). Real-time quantitative polymerase chain reaction (qPCR) reagents (\#QPK-201) were from TOYOBO (Shanghai, China). A dual-luciferase detection kit (E1910) was obtained from Promega (Madison, WI).

\section{BSBP assay}

The TERT promoter binding proteins were analyzed by streptavidin-agarose pulldown as described previously ${ }^{31}$. Briefly, the $5^{\prime}$ biotin-labeled double-stranded DNA probe corresponding to nucleotide -378 to -137 of the TERT promoter region was synthesized (Sigma-Aldrich, St. Louis, $\mathrm{MO}$ ), and nuclear protein extracts of $\mathrm{HCC}$ cells were prepared. Totally, $1 \mathrm{mg}$ nuclear protein extract, $10 \mu \mathrm{g}$ DNA probe, and $100 \mu \mathrm{l}$ streptavidin-agarose beads (SigmaAldrich) were mixed well and incubated at $4{ }^{\circ} \mathrm{C}$ overnight. The mixture was then washed to eliminate proteins bound at the DNA probe nonspecifically, centrifuged at $500 \times g$ to precipitate the TERT promoter fragment/binding protein complex. The TERT promoter fragment binding proteins were separated by sodium dodecyl sulfate-polyacrylamide gel electrophoresis (SDS-PAGE) and visualized by silver staining (Beyotime, Haimen, China). 


\section{Mass spectrometry (MS)}

The HCC specific TERT promoter binding protein band in the PAGE gel was cut out and bleached with $30 \% \mathrm{ACN} /$ $100 \mathrm{mM} \mathrm{NH}_{4} \mathrm{HCO}_{3}$. After reduction and alkylation, the proteins in the band were digested with MS-grade trypsin solution (Promega, Madison, WI) and analyzed by ultrafleXtreme ${ }^{\mathrm{TM}}$ matrix-assisted laser desorption ionizationtime of flight mass spectrometry (MALDI-TOF)/TOF mass spectrometer (Bruker, Germany).

\section{Chromatin immunoprecipitation (ChIP) assay}

ChIP assay was performed as described in Carey's protocol $^{32}$. Briefly, the cells were fixed with $1 \%$ formaldehyde, and the cross-linking was quenched by glycine (final concentration $137.5 \mathrm{mM}$ ). DNAs were sonicated on ice into $300-1000 \mathrm{bp}$ fragments. One-third of each sample was used as the DNA input control, and the remaining two-thirds were subjected to immunoprecipitation with anti-NCOA3 antibody or nonimmune rabbit IgG (Cell Signaling Technology). PCR was performed to amplify a $250 \mathrm{bp}$ TERT promoter segment. The PCR products were resolved in a $2 \%$ agarose gel and visualized by Gel-Red staining. ChIP-qPCR was performed using 9 primer pairs covering -1518 to +40 of TERT promoter (Supplementary Table 1). The relative enrichment of each fragment was normalized to the input.

\section{Electrophoretic mobility shift assay (EMSA)}

The biotin-labeled DNA probes of TERT promoter regions -234 to -144 and -696 to -456 were synthesized. The EMSA assay was performed following the standard protocol of the Pierce Light Shift kit. Briefly, the probes, HCC cell nuclear extracts, and NCOA3 antibody were incubated at $25^{\circ} \mathrm{C}$ for $20 \mathrm{~min}$ for a binding reaction. The NCOA3-probe complexes and free probes were separated in a $4 \%$ polyacrylamide gel and transferred to a nylon membrane. After ultraviolet cross-linking, the nylon membrane was treated with EMSA blocking buffer and then incubated with streptavidin-HRP conjugated solution. The bands were detected with ECL solution by Molecular Imager ChemiDoc ${ }^{\mathrm{Tm}}$ XRS + and analyzed using the Image Lab software (Bio-Rad, Hercules, CA).

\section{Promoter reporters and dual-luciferase assay}

To detect the regulation of NCOA3 on TERT promoter activity, truncation fragments of the TERT promoter ( -902 to $+40,-321$ to $+40,-234$ to $+40,-144$ to +40 , -70 to $+40,-40$ to +40 ) were amplified and inserted into SacI and HindIII sites of the firefly luciferase vector pGL4.10 (Promega, Madison, WI). and renilla luciferase reporter vector pRL-TK served as a control. The primers were shown in Supplementary Table 1. The HCC cells with NCOA3 overexpression or knockdown and the control cells were seeded into 96-well plates $\left(2 \times 10^{4}\right.$ cells/well) and transfected with pGL4.10-TERT-truncation/pRL-TK (30:1-50:1) plasmids with Lipofectamine 3000 (Invitrogen, Carlsbad, CA). At $36 \mathrm{~h}$ after transfection, cells were lysed, and the dual-luciferase assay was performed according to the introduction of the DualLuciferase ${ }^{\circledR}$ Reporter Assay System (Promega).

\section{Quantitative PCR (qPCR)}

For RT-qPCR, total RNA was isolated using TRIZOL Reagent (Invitrogen, Carlsbad, CA), and cDNA was synthesized using Rever Tra Ace qPCR RT Kit (TOYOBO \#FSQ-101, Shanghai, China) for qPCR. For ChIP-qPCR, the ChIP DNA fragments and input genomic DNAs served as temples. qPCR was performed with the SYBR Green PCR master mix (Applied Biosystems, Waltham, MA), and the amplification signals were detected by CFX96 Touch ${ }^{\mathrm{TM}}$ (Bio-Rad, Hercules, CA) and analyzed by CFX Manager 3.0 (Bio-Rad). Target gene relative expression level was calculated by $2^{-\Delta \mathrm{CT}}(\Delta \mathrm{CT}=$ $\left.\mathrm{CT}_{\text {Target gene }}-\mathrm{CT}_{\mathrm{GAPDH}}\right)$ and normalized to the relative expression level detected in control cells. Each sample was tested in triplicate.

\section{Western blot}

The HCC specimens and cells were homogenized with RIPA lysis buffer (Beyotime, Haimen, China) or Complete Lysis-M reagent (Roche, Indianapolis, IN) supplemented with cocktail protease inhibitors (Roche). The Protein concentration was detected by BCA assay (Pierce, Waltham, MA), and the proteins were separated by SDSPAGE and transferred to polyvinylidene difluoride membranes. The membranes were blocked and sequentially incubated with primary antibodies and HRPconjugated secondary antibody. The proteins were detected using an ECL solution.

\section{Lentivirus production and cell transfection}

To establish HCC cells with stable NCOA3 overexpression, we constructed lentivirus pLV[Exp]-PuroCMV-NCOA3-IRES:EGFP which encoded a full-length human NCOA3 gene, and PLV-CMV-EGFP-PGK-Puro as empty vector control. The lentivirus or the empty vector were transfected into 293FT cells together with lentivirus packing plasmids (pMDL:VSV-G:REV $=5: 3: 2$ ) for NCOA3 lentivirus packing. The virus was then harvested to infect HCC cells, and stable clones with NCOA3 overexpression were selected after 2 weeks with $0.7-2 \mu \mathrm{g} / \mathrm{ml}$ puromycin. The expression of NCOA3 in the clones was determined by RT-qPCR and western blot. The hU6-NCOA3-EGFP-IRES-puromycin and negative control lentiviral vector containing non-silencing short hairpin RNA lentivirus plasmid was obtained from Genechem Company Ltd. (Shanghai, China). Two target sequences (5'-GCGCCAGAGATATGAAACA-3' and 5'- 


\section{GGCAG}

GGAGTTATTGATAA-3') were used to knockdown NCOA3 expression. The efficiency of RNA interference was determined by RT-qPCR and Western Blot.

\section{Cell viability assay}

Cells were seeded in 96-well plates $\left(2 \times 10^{3}\right.$ cells/well $)$. The viability of HCC cells was assessed by MTS assay (Promega, Madison, WI). The absorbance at $490 \mathrm{~nm}$ of each well was measured with a microplate reader. Each group included six replicates and three independent experiments were performed.

\section{Colony formation assay}

Cells were seeded in 6-well plates (100-1000 cells/well) and cultured for 1-2 weeks. The colonies were fixed with $4 \%$ paraformaldehyde for $15 \mathrm{~min}$ at room temperature, stained with $2 \%$ crystal violet for $30 \mathrm{~min}$, and counted under a microscope. Three independent experiments were performed.

\section{In vivo xenograft experiments}

All animal procedures were performed following the Guide for the Care and Use of Laboratory Animals (NIH publication Nos. 80-23, revised 1996) and the Institutional Ethical Guidelines for Animal Experiments developed by Sun Yat-Sen University. Totally, 4-5-week old female SPF $\mathrm{BALB} / \mathrm{c}$ nude mice were obtained from Beijing Vital River Laboratory Animal Technology Co., Ltd. The mice were subcutaneously injected with $3 \times 10^{6} \mathrm{Hep} 3 \mathrm{~B}$ cells $(5$ mice for each group). The length $(L)$, width $(W)$, and height $(H)$ of the tumors were measured with calipers every 4 days, and the tumor volume $(V)$ was calculated as $V=\pi L W H / 6$. The standard streptavidin-biotin-peroxidase complex method was used for tumor xenograft immunohistochemical (IHC) staining. Briefly, after deparaffinization, blocking, and antigen retrieval, the tumor sections were incubated in a 1:100 dilution of NCOA3 or TERT-specific antibody at $4{ }^{\circ} \mathrm{C}$ overnight in a humidified chamber. After washing, tumor sections were incubated with HRP-conjugated anti-goat antibody (DakoCytomation, Carpentaria, CA) for $30 \mathrm{~min}$ at room temperature. Finally, 3,5-diaminobenzidine (DAB) substrate was used for color development, followed by Mayer's hematoxylin counterstaining.

\section{IHC assay}

HCC tissue microarray was purchased from Shanghai Outdo Biotech Co., Ltd. (OD-CT-DgLiv01-012), which contained $55 \mathrm{HCC}$ tissues and 45/55 HCC patients and had follow-up outcomes. The other 12 HCC patients from Sun Yat-Sen University Cancer Center also were included to analyze clinical outcomes. Formalin-fixed tumor specimens were deparaffinized in xylene (5 min, twice), sequentially rehydrated in $100 \%, 95 \%, 80 \%$, and $70 \%$ alcohols and distilled water. The slides were treated with $3 \% \mathrm{H}_{2} \mathrm{O}_{2}$ for $15 \mathrm{~min}$ and antigen retrieval was performed in sodium citrate buffer $(10 \mathrm{mM}$ sodium citrate, $0.05 \%$ Tween 20, pH 6.0) at $121^{\circ} \mathrm{C}$ for $5 \mathrm{~min}$. The slides were then blocked with $5 \%$ bovine serum albumin (BSA) for $30 \mathrm{~min}$ and incubated with primary antibodies overnight. After three washings with phosphate-buffered saline (PBS), the slides were incubated with HRP-conjugated secondary antibody for $30 \mathrm{~min}$, treated with $\mathrm{DAB}$ for color development, and counterstained with hematoxylin. For IHC scores, the percentage $(0-100 \%)$ of stained tumor cells was multiplied by the intensity $(0,1,2$, or 3$)$ to achieve a score between 0 and 300. All microscopy analyses were conducted by blinded experimenters.

\section{Co-immunoprecipitation (Co-IP) assay}

To investigate the interaction between NCOA3 and transcription factors in HCC cells, Co-IP assay was performed. Totally, $5 \times 10^{6}$ HCC cells were harvested, washed three times with cold PBS, and treated with Hypotonic Buffer (20 mM Tris- $\mathrm{HCl}, \mathrm{pH} 7.4,10 \mathrm{mM} \mathrm{NaCl}$, $3 \mathrm{mM} \mathrm{MgCl} 2 \cdot 6 \mathrm{H}_{2} \mathrm{O}$ ) for $15 \mathrm{~min}$ on ice. Totally, $10 \% \mathrm{NP} 40$ was added and vortexed for $15 \mathrm{~s}$. The cells were pelleted and treated with cell extraction buffer $(100 \mathrm{mM}$ Tris, $\mathrm{pH}$ 7.4, $2 \mathrm{mM} \mathrm{Na}_{3} \mathrm{VO}_{4}, 100 \mathrm{mM} \mathrm{NaCl}, 1 \%$ Triton X-100, $1 \mathrm{mM}$ EDTA, $10 \%$ glycerol, $1 \mathrm{mM}$ EGTA, $0.1 \%$ SDS, $1 \mathrm{mM} \mathrm{NaF}, 0.5 \%$ deoxycholate, $20 \mathrm{mM} \mathrm{Na}_{4} \mathrm{P}_{2} \mathrm{O}_{7} \cdot 10 \mathrm{H}_{2} \mathrm{O}$ ) supplemented with cocktail protease inhibitors in ice for $30 \mathrm{~min}$. After centrifugation, the supernatant was collected and pre-cleared with $50 \%$ protein $\mathrm{A} / \mathrm{G}$ agarose beads (Millipore, Billerica, MA) for $1 \mathrm{~h}$. Primary antibody was then added in for incubation overnight, and new protein A/G agarose beads were used to pull down proteins interacting with NCOA3.

\section{IF staining}

Cells were fixed with 100\% methanol (pre-chilled at $-20^{\circ} \mathrm{C}$ ) at room temperature for $5 \mathrm{~min}$ and permeabilized with PBS containing $0.2 \%$ Triton X-100 for $10 \mathrm{~min}$. After three 5-min washings with PBS, the cells were blocked with $1 \%$ BSA and $22.52 \mathrm{mg} / \mathrm{ml}$ glycine in PBST for $30 \mathrm{~min}$ and incubated with primary antibodies in PBST containing $1 \%$ BSA in a humidified chamber overnight at $4{ }^{\circ} \mathrm{C}$. After three washings with PBS, fluorescence-conjugated secondary antibodies in PBST containing 1\% BSA were added to the cells and incubated for $1 \mathrm{~h}$ at room temperature in the dark. NCOA3, TERT, and SP1 expression in HCC cells were observed under a confocal microscope.

\section{Statistical analysis}

Data were presented as the mean \pm standard deviation of at least three independent experiments. Statistical analysis was carried out using SPSS 11.0 software (SPSS 
Inc., Chicago, IL). Statistical tests were justified as appropriate. $P<0.05$ was considered significant.

\section{Results}

NCOA3 was identified as a TERT promoter binding protein in HCC cells

The transcription of TERT was over-activated in HCC cells. In order to discover and identify the specific transcriptional factors that regulate TERT expression and cell growth in HCC, we used the BSBP method coupled with MALDI-TOF/TOF MS approach to identify the TERT promoter binding proteins in HCC cell lines (HepG2, Hep3B, BEL-7402, SNU-449) and immortalized liver cell line (LO2). The non-liver cancer cell line (HEK293) was used as a negative control. We found that NCOA3, a potential candidate, differentially bound to the TERT promoter in HCC cells (Fig. 1A, Fig. S1A). To validate that NCOA3 bound to the TERT promoter, we detected NCOA3 with its specific antibody in the nuclear proteins pulled down by the TERT promoter probe in LO2 immortalized liver cells and HCC cells. As is shown in Fig. $1 \mathrm{~B}$, a higher level of NCOA3 was pulled down in the HepG2, SNU-449, BEL-7402, and Hep3B HCC cells than in LO2 cells. Moreover, a ChIP assay was performed to confirm that NCOA3 bound to TERT promoter in LO2 and HCC cells, and the results showed that NCOA3 had much stronger binding at the endogenous TERT promoter in HCC cells compared to LO2 cells (Fig. 1C). We detected the mRNA expression of TERT in HCC cells and LO2 cells and found that TERT expression was higher in HCC cells than in LO2 cells (Fig. S1B). Consistently, the expression of NCOA3 was higher in HCC cells (Fig. 1D).

Next, to identify the exact binding locus of NCOA3 at the TERT promoter, we performed ChIP-qPCR with nine pairs of primers that covered the $1500 \mathrm{bp}$ region upstream of the TERT transcription start site (TSS). Our results showed that NCOA3 was enriched at the -234 to -144 and -696 to -456 regions of TERT promoter in Hep3B cells (Fig. 1E), and EMSA assay confirmed that NCOA3 bound to the -234 to -144 region of TERT promoter (Fig. 1F). Accordingly, these data validated NCOA3 as a new TERT promoter binding protein in HCC cells.

\section{NCOA3 transcriptionally activated TERT expression in HCC cells}

To test whether NCOA3 bound to the TERT promoter to activate TERT transcription, we established HCC cell lines with stable knockdown or overexpression of NCOA3 by lentivirus infection. We found that knockdown of NCOA3 decreased TERT mRNA and protein levels in HepG2 and Hep3B cells (Fig. 2A, B), while overexpression of NCOA3 enhanced TERT expression in SNU-449 and Hep3B cells (Fig. 2F). To examine whether NCOA3 regulated TERT promoter activity, we constructed a series of luciferase reporter plasmids that contained different segments of the -902 to +40 region of TERT promoter. Our results indicated that the -234 to -40 region was essential for potent TERT promoter activity (Fig. 2C, shCtrol). Also, knockdown of NCOA3 dramatically decreased the promoter activity of the -234 to +40 and -144 to +40 fragments, but not the activity of the -70 to +40 fragment (Fig. 2C), which suggested that the -234 to -70 region of TERT promoter contained a major NCOA3-binding site. This was in accordance with our ChIP-qPCR and EMSA results (Fig. 1E, F). We further confirmed the role of NCOA3 in regulating the activity of TERT promoter in HCC cells with the luciferase reporter plasmid that had the -234 to +40 fragment. Knockdown of NCOA3 significantly decreased the TERT promoter activity in Hep3B and HepG2 cells (Fig. 2D). In contrast, overexpression of NCOA3 remarkably enhanced the TERT promoter activity in SNU-449 and Hep3B cells (Fig. $2 \mathrm{E}, \mathrm{F})$. Taken together, these data indicated that NCOA3 bound to the TERT promoter and activated TERT transcription.

\section{NCOA3 promoted tumor cell growth via TERT signaling in $\mathrm{HCC}$ cells and mouse HCC xenograft model}

To investigate the function of NCOA3 in HCC cell growth, we first established SNU-449 and Hep3B cells with stable NCOA3 overexpression. As shown in Fig. 3A, $B$, overexpression of NCOA3 effectively enhanced HCC cell viability and colony formation. Moreover, overexpression of NCOA3 increased the size and weight of tumors in a mouse HCC xenograft model (Fig. 3C, D), though the bodyweight of the mice remained unchanged (Fig. 3E). IHC staining showed that the expression of TERT was elevated in xenograft tumors with NCOA3 overexpression (Fig. 3F).

Next, we established HepG2 and Hep3B cells with stable knockdown of NCOA3 and found that NCOA3 knockdown significantly inhibited HCC cell viability and colony formation (Fig. 4A, B). Interestingly, overexpression of TERT partly rescued the inhibition of cell viability and colony formation caused by NCOA3 knockdown (Fig. 4A, B), which suggested that NCOA3 promoted HCC cell growth via TERT signaling. Consistently, results from our HCC xenograft model showed that NCOA3 knockdown decreased the size and weight of tumors without affecting the mouse body weight, and the decrease was partly reversed by TERT overexpression (Fig. 4C, E). Also, IHC staining showed that the expression of TERT was decreased in xenograft tumors when NCOA3 was knocked down (Fig. 4D).

It is known that TERT activates Wnt signaling independently of its telomerase reverse transcriptase activity ${ }^{27}$. To further examine whether NCOA3 promoted HCC growth via the TERT signaling pathway, we examined the 
A

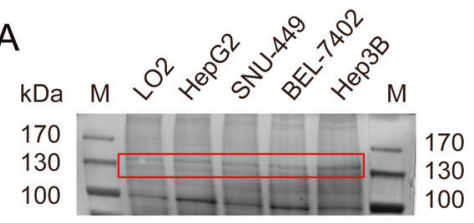

C

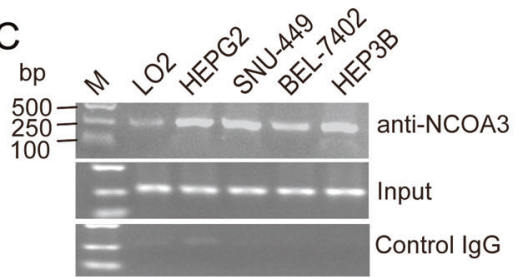

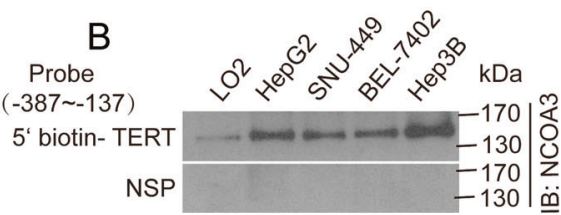

$\mathrm{D}$
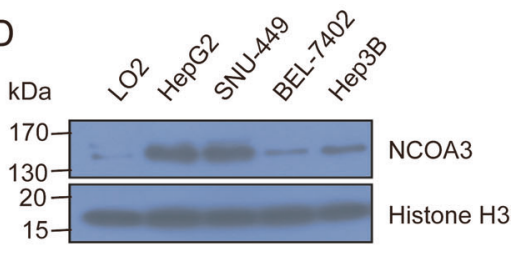
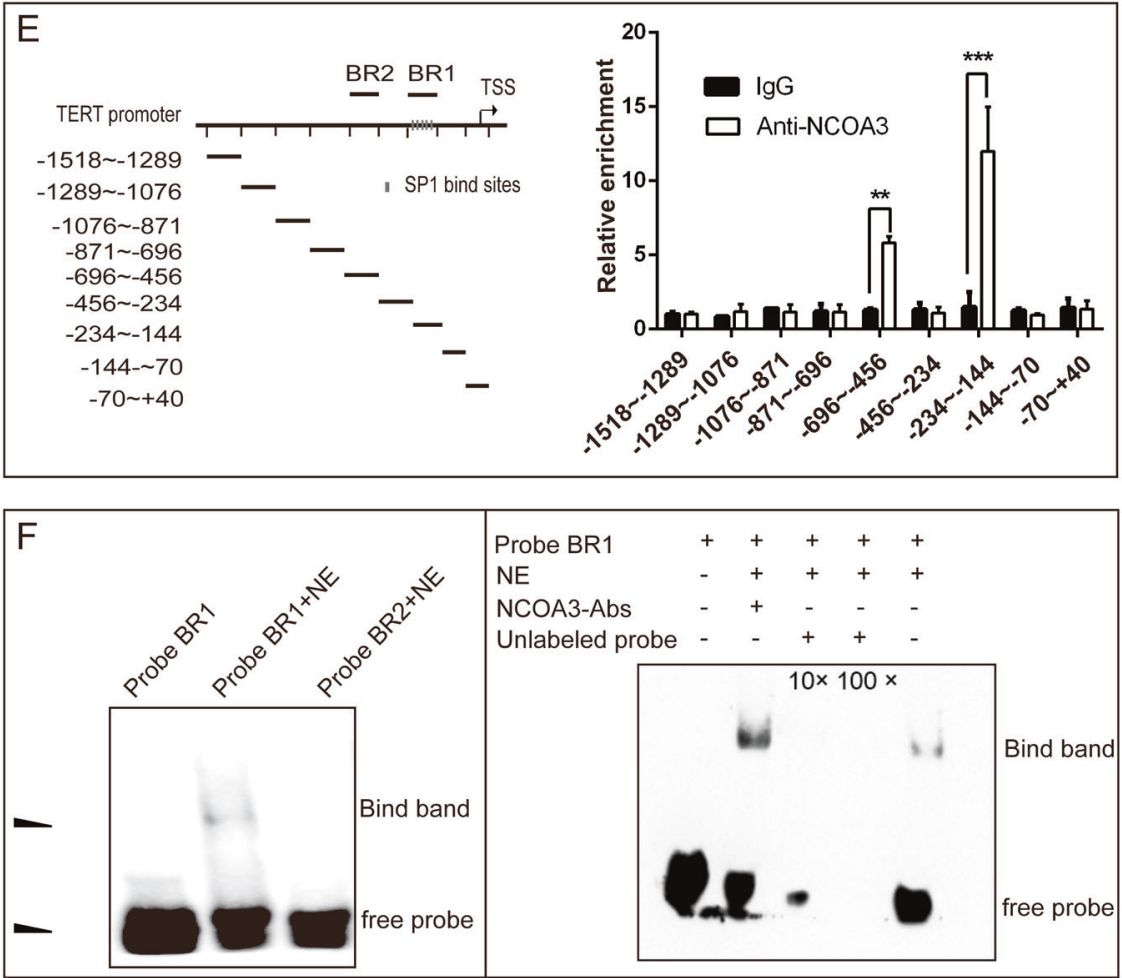

Fig. 1 NCOA3 was identified as a new TERT promoter binding protein in HCC cells. A Potential TERT promoter binding proteins were pulled down using a $5^{\prime}$ biotin-labeled TERT promoter ( -387 to -137) double-stranded DNA probe and streptavidin-beads in four HCC cell lines (HepG2, SNU-449, BEL-7402, and Hep3B) and one immortalized liver cell line (LO2). The proteins were separated by SDS-PAGE and visualized by silver staining. The box indicated the protein band that was enriched in HCC cells and processed for MS analysis. B NCOA3 was detected in the pull-down mixture by the $5^{\prime}$ biotin-labeled TERT promoter probe or a nonspecific probe (NSP) by western blot. C ChIP assay was performed in LO2 and HCC cells with NCOA3 antibody and TERT promoter-specific primers. The PCR products were separated in a $2 \%$ agarose gel, and lgG was used as a negative control. D Protein level of NCOA3 in the nuclear extracts of $\mathrm{LO} 2$ and HCC cells. Histone $\mathrm{H} 3$ served as a control. E lllustration of the primer pairs for the ChIPQPCR assay. BR1 binding region 1, BR2 binding region 2, TSS transcription start site. Enrichment of NCOA3 at different regions of TERT promoter was detected by ChIP-qPCR in Hep3B cells. Data were shown as means \pm SD. ${ }^{* *} P<0.01 .{ }^{* *} P<0.001$, Student's $t$ test. Each sample was tested in triplicate. F EMSA assay was performed with $5^{\prime}$ biotin-labeled TERT promoter probe BR1 ( -234 to -144$)$ or BR2 ( -696 to -456$)$ and the nuclear extract of Hep3B cells. The upper arrowhead denoted the probes bound by NCOA3, and the lower arrowhead denoted free probes. NE nuclear extract. The supper shift assay was performed using NCOA3 specific antibody, BR1, and the nuclear extract of Hep3B cells. Unlabeled TERT promoter probes were added as the competitor.

expression of the Wnt target genes in NCOA3 knockdown Hep3B cells and found that knockdown of NCOA3 decreased the expression of the Wnt targets MYC, TCF-1, and CCND1, but had no effect on the expression of the
Notch target genes HES1, HES6, HEY1, and NRARP (Fig. 4F). Collectively, these in vitro and in vivo results demonstrated that NCOA3 promoted HCC cell growth via TERT signaling. 


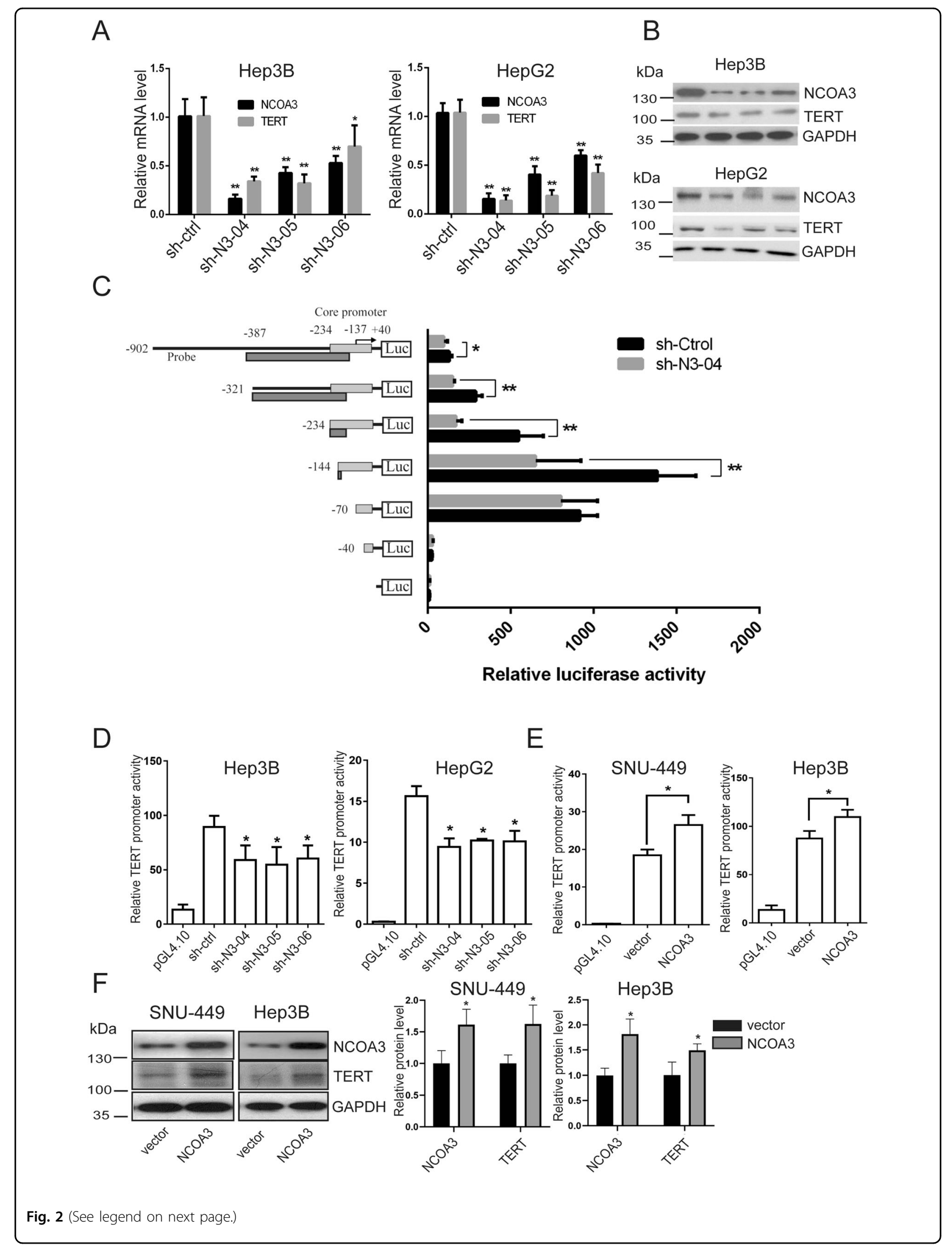




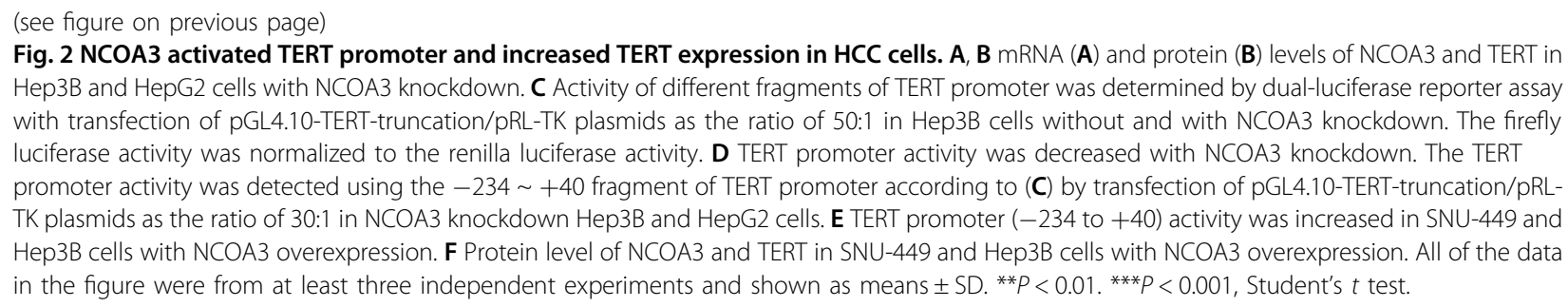

NCOA3 cooperated with SP1 to promote TERT expression and HCC cell growth

As a transcriptional coactivator, NCOA3 interacts with many bHLH (basic helix-loop-helix) transcription factors like HIF1 $\alpha$ through its N-terminal domain to activate target gene expression ${ }^{22,28}$. In order to identify the exact transcription factor(s) that interacted with NCOA3 to activate TERT transcription, we analyzed the transcription factors binding to the $-300 \sim$ +68 region of TERT promoter and found that this region had many SP1-binding sites (Fig. 5A). Considering that SP1 is a bHLH domain-containing transcription factor, and it is critical for TERT transcription activation, we hypothesized that NCOA3 interacted with SP1 to activate TERT transcription and promote HCC cell growth. Endogenous Co-IP experiment revealed that NCOA3 interacted with SP1 in Hep3B and HepG2 cells, and this interaction was enhanced by NCOA3 overexpression (Fig. 5B). Also, SP1 was detected on the $5^{\prime}$ biotin-labeled TERT promoter probe (Fig. 1B) pulled down by NCOA3 antibody in $\mathrm{HCC}$ cells (Fig. 5C). In addition, immunofluorescence (IF) showed that NCOA3 was co-localized with SP1 in the nuclei of Hep3B and HepG2 cells (Fig. 5D). We further explored whether NCOA3 and SP1 coregulated TERT expression and found that knockdown of SP1 in Hep3B cells abolished the TERT expression induced by NCOA3 overexpression (Fig. 5E). Consistently, knockdown of SP1 partially inhibited the growth of Hep3B cells caused by NCOA3 overexpression (Fig. 5F). Taken together, these results indicated that NCOA3 interacted with SP1 to promote TERT expression and HCC cell growth.

\section{The expression of NCOA3 and TERT were elevated in HCC tissues}

Analysis of the TCGA database for TERT gene variations in liver cancer patients revealed that TERT gene amplification occurred in about 4\% (23/587) of the liver cancer patients (Fig. S2A). However, TERT mRNA was upregulated in over half (338/587) of the patients (Fig. S2B), suggesting that the increased expression of TERT in HCC was caused by elevated TERT transcription. We then detected the NCOA3 and TERT protein expression in the tumor and peritumoral tissues of $30 \mathrm{HCC}$ patients from the Sun Yat-Sen University Cancer Center. Our results showed that both NCOA3 and TERT had higher expression in the tumor tissues (Fig. 6A, B), and the expression of NCOA3 and TERT were, respectively, elevated in $88 \%(26 / 30)$ and $78 \%$ (23/30) of the HCC tissues. Moreover, the expression of TERT was positively correlated with the expression of NCOA3 in HCC patients ( $r=$ 0.391, $p=0.002$ ) (Fig. S2C). Consistently, NCOA3 and TERT had higher expression in HCC cells (BEL-7402, SNU-387, SNU-449, Hep3B, and HepG2) compared with the LO2 immortalized liver cells (Fig. 6C). We also analyzed the correlation between NACO3, SP1 and TERT mRNA expression in liver cancer patients in the GEO database (GEO: GSE10143), the mRNA level of SP1 was a positive correlation with the NCOA3 $\left(R^{2}=0.7367, p<\right.$ $0.0001)$ and TERT $\left(R^{2}=0.0 .401, p<0.0001\right)$ (Fig. S2D). Moreover, the Kaplan-Meier survival analysis of GEO data showed that the patients with high mRNA expression of SP1, NACO3, and TERT had significantly shorter survival times (Fig. S2E, F). These results indicated that the expression of SP1, NACO3, and TERT had a positive correlation in tumor tissues. Moreover, the combination of the expression of SP1, NACO3, and TERT may serve as a survival predictor in hepatocellular carcinoma.

\section{High expression of NCOA3, SP1, and TERT was associated with poor clinical outcomes in HCC patients}

We further analyzed the clinical significance of NCOA3/TERT high expression in HCC patients. Tissue microarray data showed that the expression of NCOA3 and TERT were positively correlated in HCC patients $(n=53)$ (Fig. 6D, E), and were higher in tumor tissues than in adjacent nontumor tissues (Fig. 6F). Moreover, we analyzed the follow-up outcome data from the 45 patients in the tissue microarray and 12 other $\mathrm{HCC}$ patients (Fig. $6 \mathrm{~A}, \mathrm{~B})$, and found that the HCC patients $(n=57)$ with high expression of NCOA3(16/57) or TERT (12/57) had poor prognosis (Fig. 6G, H), and the patients with high expression of both NCOA3 and TERT(6/57) had the worst outcomes, whereas the patients with low expression of both NCOA3 and TERT (35/57) had the best outcomes 


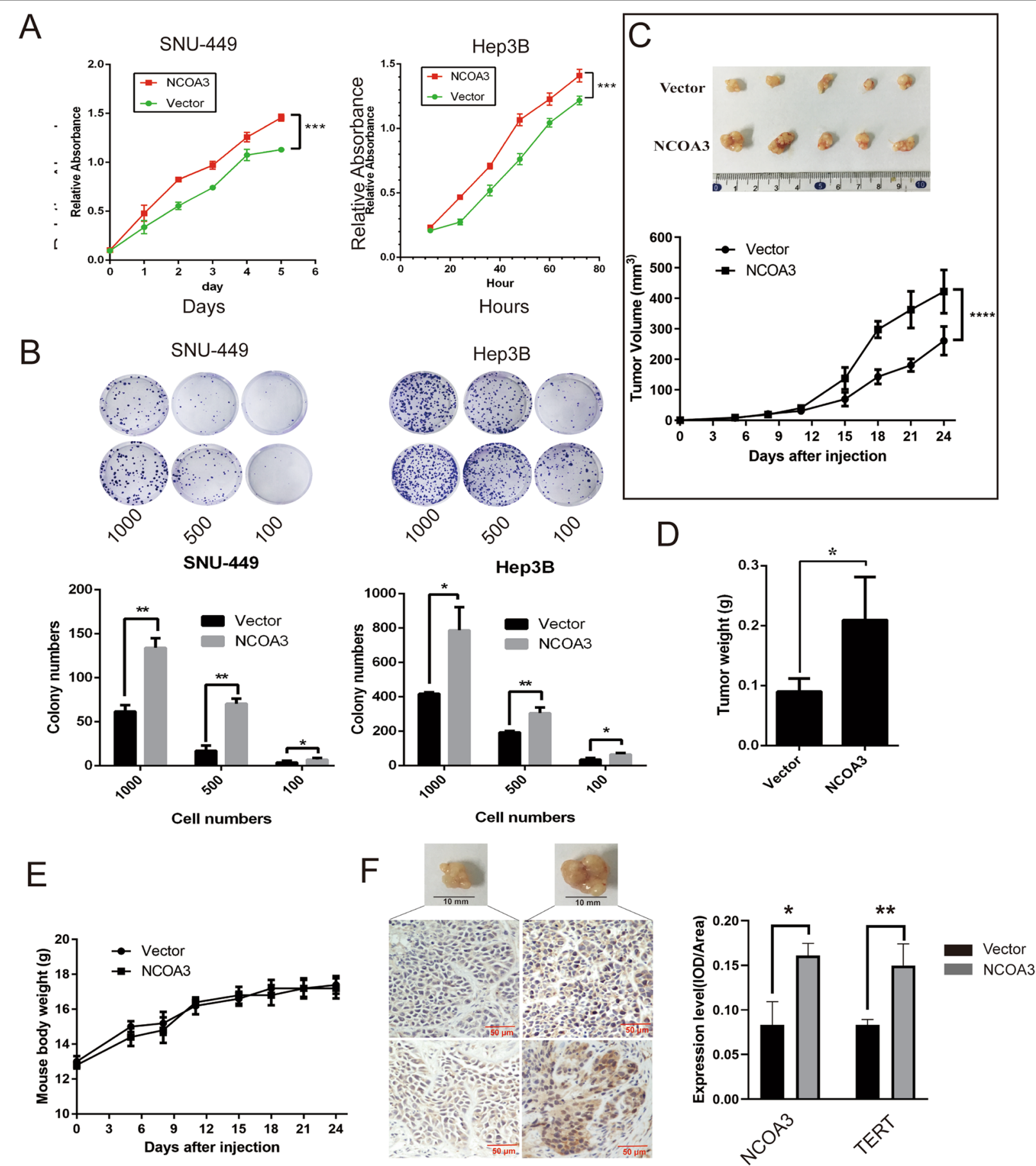

Fig. 3 Overexpression of NCOA3 promoted HCC cell growth in vitro and in vivo. A Viability of SNU-449 and Hep3B cells with NCOA3 overexpression was determined by MTS assay. B The colony formation ability of SNU-449 and Hep3B cells with NCOA3 overexpression. The colonies were stained by crystal violet and counted. C Nude mice were subcutaneously injected with Hep3B cells with vector or NCOA3 overexpression. Images of the HCC tumor xenograft from each mouse ( $n=5$ mice/group) were captured, tumor volumes were recorded every 3 days, $\mathbf{D}$ tumor weights were analyzed, $\mathbf{E}$ mouse body weights were monitored, and $\mathbf{F}$ the expression of NCOA3 and TERT in tumor tissues were detected by IHC staining. The data were shown as means $\pm \mathrm{SD},{ }^{*} P<0.05$, ${ }^{* *} P<0.01$. ${ }^{* *} P<0.001$, Student's $t$ test.

(Fig. 6I). Furthermore, we also analyzed the prognosis of the 156 liver cancer patients with high and low expression of NCOA3/TERT/SP1 in the clinic date sets of the GEO database (GSE10143) and found the patients with high expression with NCOA3, SP1, or TERT have a poor prognosis comparison the HCC patients with low 


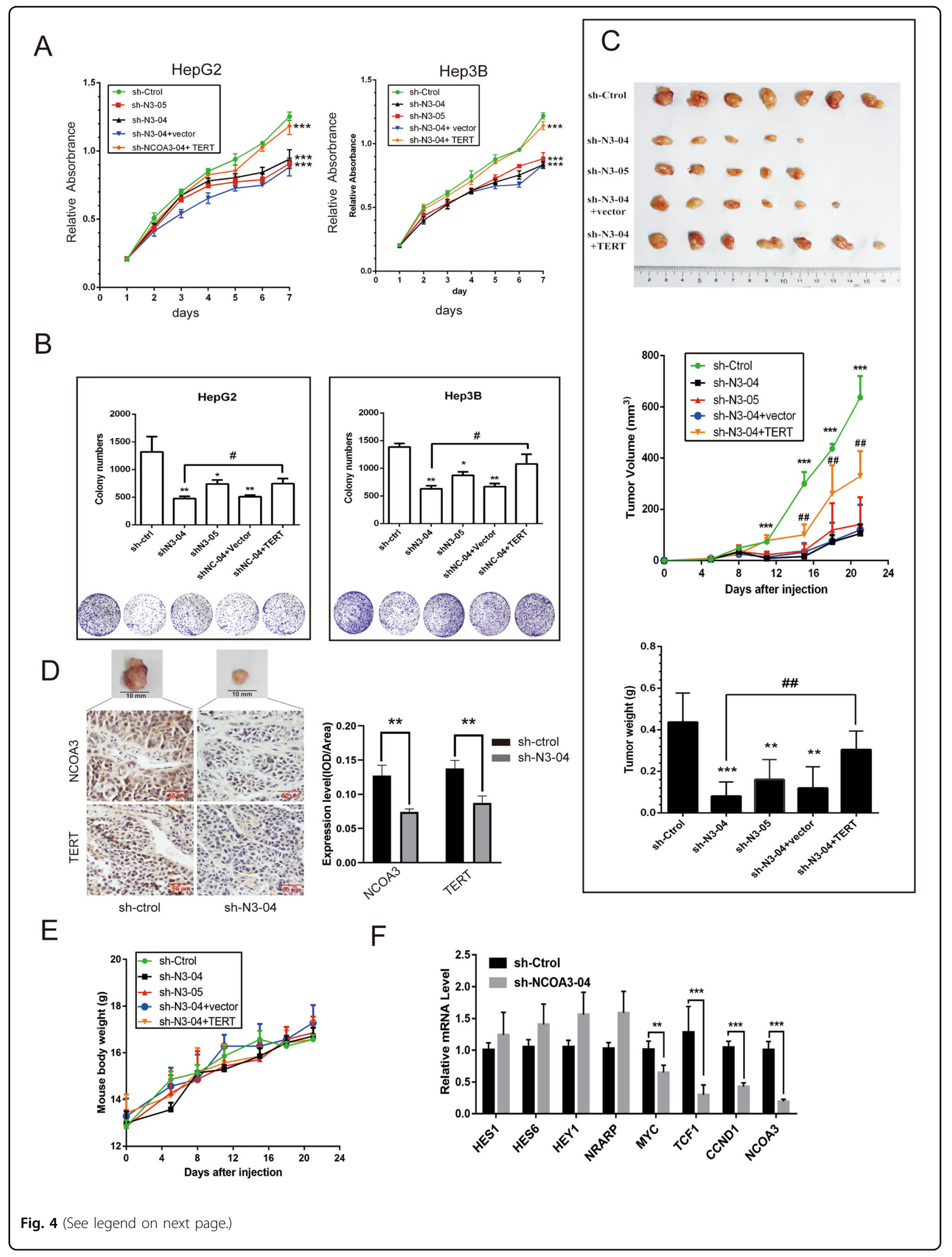


(see figure on previous page)

Fig. 4 Knockdown of NCOA3 inhibited HCC cell growth in vitro and in vivo. A Viability of HepG2 and Hep3B cells with NCOA3 knockdown. B The colony formation ability of HepG2 and Hep3B cells with NCOA3 knockdown. C Nude mice were subcutaneously injected with Hep3B cells with nonspecific shRNA (sh-Ctrol), NCOA3-specific shRNAs (sh-N3-04, sh-N3-05), sh-N3-04 + vector, or sh-N3-04 + TERT overexpressing plasmid (TERT). Images of the HCC tumor xenograft from each mouse ( $n=5$ mice/group) were captured, tumor volumes were recorded every 4 days, tumor weights were analyzed, $\mathbf{D}$ the expression of NCOA3 and TERT in tumor tissues were detected by $\mathrm{HHC}$ staining, and mouse body weights were monitored (E). F mRNA levels of the Notch target genes (HES1, HES6, HEY1, and NRARP), Wnt target genes (MYC, TCF-1, and CCND1), and NCOA3 in Hep3B cells with NCOA3 knockdown were measured by RT-qPCR. The data were shown as means \pm SD, ${ }^{*} P<0.05$, ${ }^{* *} P<0.01$. ${ }^{* *} P<0.001,{ }^{\#} P<0.05$, ${ }^{\# \#} P<0.01$,

Student's $t$ test.
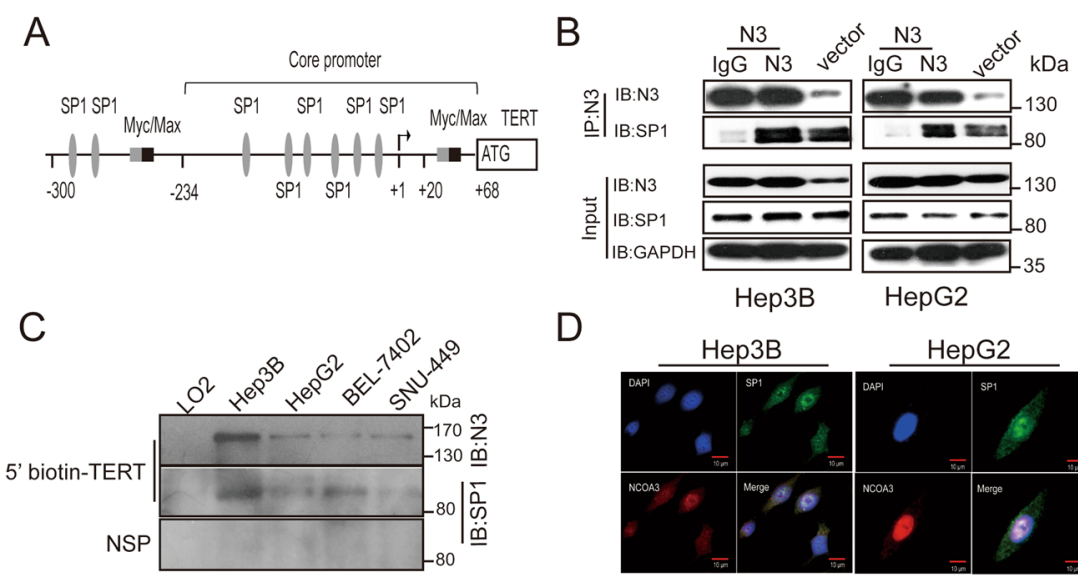

$\mathrm{D}$

Hep3B HepG2
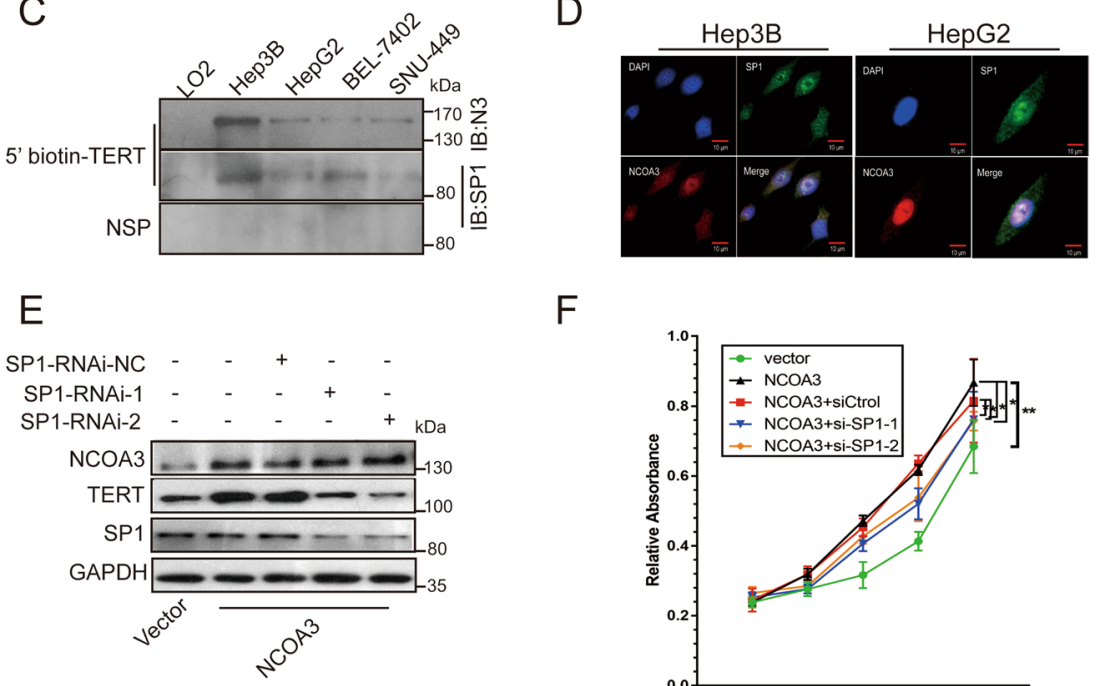

F

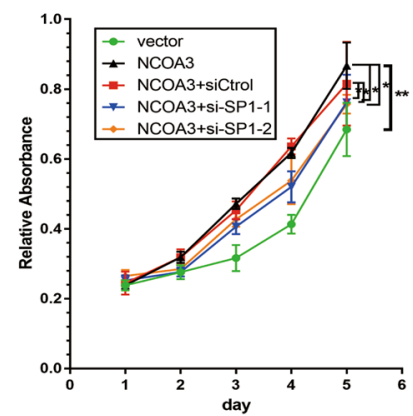

Fig. 5 NCOA3 interacted with SP1 to promote TERT expression and HCC cell growth. A Illustration of the SP1 binding sites in the TERT promoter. B The interaction between NCOA3 and SP1 was tested by co-IP in Hep3B and HepG2 cells without (vector) or with NCOA3 overexpression (N3). NCOA3 antibody was added to the nuclear extracts to precipitate proteins interacting with NCOA3, while $\lg G$ was used as a negative control. SP1 was then detected by its specific antibody in the precipitates. C The $5^{\prime}$ biotin-labeled TERT promoter probe was pulled down by NCOA3 antibody in immortalized LO2 and HCC (Hep3B, HepG2, BEL-7402, and SNU-449) cells, while a nonspecific probe (NSP) was used as a negative control. SP1 was detected by its specific antibody in the precipitates. D Immunofluorescence of NCOA3 (red) and SP1 (green) in Hep3B and HepG2 cells. E TERT expression was detected in Hep3B cells without (Sp1-RNAi-NC) or with SP1 knockdown (Sp1-RNAi-1, Sp1-RNAi-2). F Viability of Hep3B cells with NCOA3 overexpression and SP1 knockdown. The data were shown as means \pm SD, ${ }^{*} P<0.05,{ }^{* *} P<0.01$, Student's $t$ test from three independent experiments.

expression of this gene (Fig. S2E). And, the HCC patients with both high expression of SP1/NCOA3 and SP1/TERT have the worst prognosis compared with those with low SP1, NCOA3, and TERT expression (Fig. S2F).

Taken together, these results suggested that high expression of NCOA3/TERT/SP1 was a key factor to predict the prognosis of HCC patients.

\section{Discussion}

Our study has for the first time identified NCOA3 as a transcriptional coactivator that bound to the -234 to -144 region of TERT promoter in HCC cells, regulated TERT expression through interaction with SP1, and thus promoted HCC cell growth via TERT signaling. We have also found that NCOA3/TERT high 


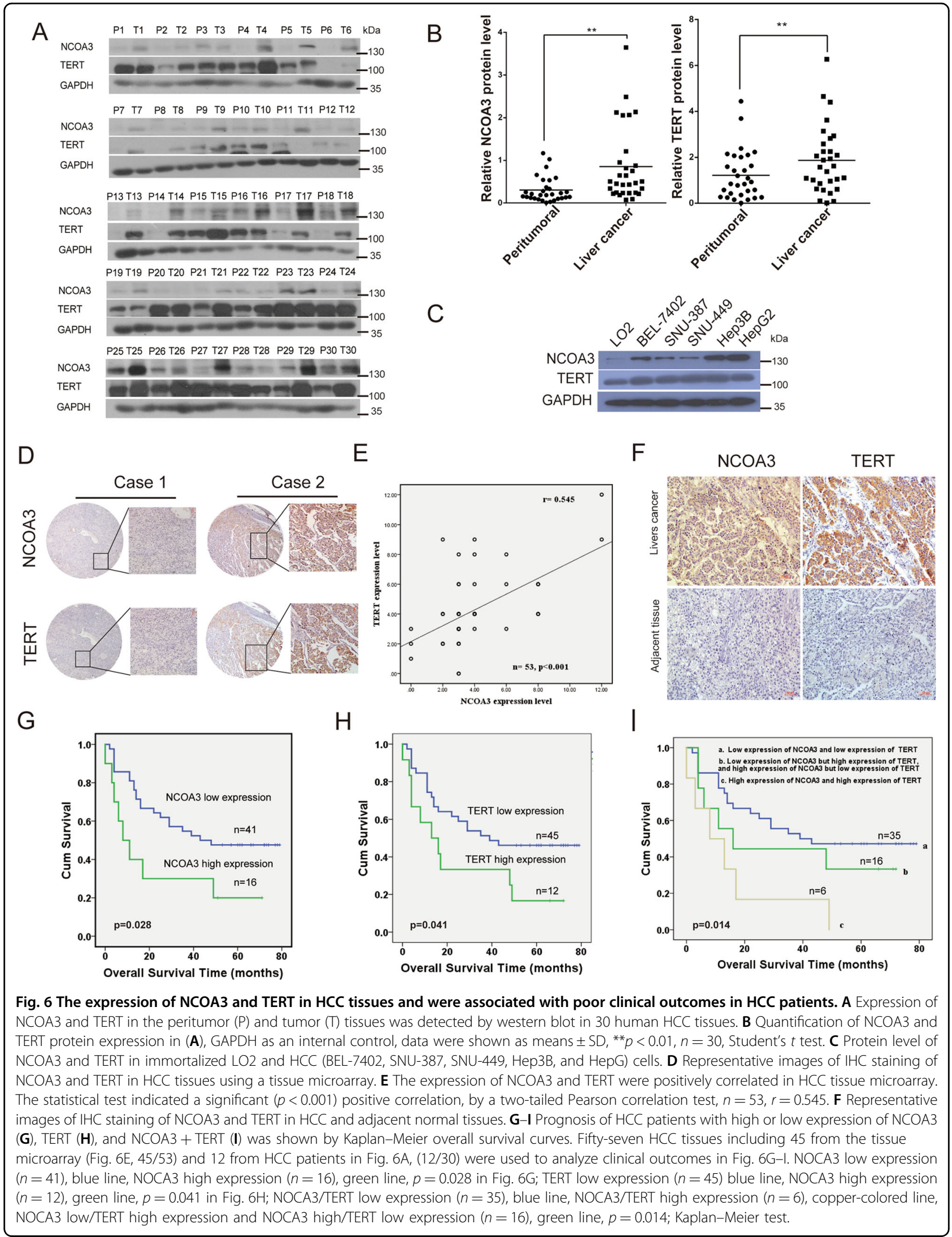


expression in HCC tissues is a poor prognosis factor for HCC patients. Our findings have revealed a novel role of NCOA3 in the regulation of TERT expression and HCC tumorigenesis and suggested that it could be a target for HCC therapy.

TERT is the catalytic subunit of telomerase that maintains the length of telomeres and thus plays a decisive role in cell immortalization ${ }^{33,34}$. In recent years, TERT has been reported to mediate many molecular events independently on its reverse transcriptase activity, which include activation of the $\mathrm{Wnt} / \beta$-catenin signaling, induction of angiogenesis, regulation of DNA damage response, and inhibition of apoptosis in cancer development ${ }^{35-40}$. Therefore, TERT is regarded as a central regulator of the hallmarks of cancer, and therapeutics targeting both its canonical and noncanonical functions are suggested to be more effective in cancer treatment ${ }^{9}$.

Our analysis of TERT gene variations in liver cancer patients using the TCGA database revealed that amplification of the TERT gene occurred in only $~ 4 \%(23 / 587)$ of the liver cancer patients (Fig. S2A), which was consistent with the studies by other groups ${ }^{30,41}$. In contrast, the mRNA level of TERT was upregulated in over 55\% (338/ 587) of the liver cancer patients (Fig. S2B), suggesting that the expression of TERT was mainly regulated on the transcription level. The TERT gene consists of 15 introns and 16 exons, and the TERT core promoter is located in -234 upstream of its TSS to +37 of the second exon ${ }^{42}$, containing enriched CpG dinucleotide sites, SP1 binding sites, and E-box, but lacks TATA box and CAAT box ${ }^{43}$. The transcription of TERT is regulated by many positive and negative factors, and SP1 and Myc are two common transcription factors that activate TERT expression in cancer cells ${ }^{44,45}$. SP1 is a zinc finger transcription factor that binds to the GC-rich region of TERT promoter ${ }^{46}$, while Myc belongs to the bHLH-zip (basic helix-loop-helix-zipper) protein family and forms a heterodimer with Max to bind at the E-box of TERT promoter ${ }^{47}$. Studies have suggested that the elevated TERT expression mediated by Myc/Max depends on SP1 ${ }^{45}$. In this study, we discovered that NCOA3 bound to the -234 to -144 region of TERT promoter and activated TERT transcription in HCC cells (Figs. 1 and 2). Our further investigation showed that NCOA3 interacted with the transcription factor SP1 as a coactivator to promote TERT expression and HCC growth (Fig. 5).

NCOA3 is a nuclear receptor coactivator, and it interacts with other transcription factors via its $\mathrm{N}$-terminal bHLH-PAS (basic helix-loop-helix-PER-ARNT-SIM) domain ${ }^{48}$. The bHLH-PAS family proteins are important in several protumor and antitumor pathways ${ }^{49}$. In this study, we found that NCOA3 had higher expression in HCC cells than in the LO2 immortalized hepatocytes (Fig. 6C), and in HCC tumor tissues than in peritumoral tissues (Fig. 6A, B). Moreover, our results showed that high expression of NCOA3 promoted TERT expression and $\mathrm{HCC}$ cell growth (Fig. 2 and Fig. 3). Considering that SP1 is a general transcription factor and interacts with bHLH proteins, we propose that the elevated expression of NCOA3 and its interaction with SP1 is crucial to the transcription activation of TERT in HCC. In addition, NCOA3 can recruit the acetyltransferase p300/CBP through its $\mathrm{AD}$ at the $\mathrm{C}$-terminus ${ }^{48}$, so we speculate that NCOA3 may also activate TERT transcription by recruiting p300/CBP to make chromatin DNA more accessible to the RNA polymerase II transcription machinery.

Spatial and temporal modification and transporting of essential regulators between different cell compartments is one of the key events in cellular signal transduction, gene activation, and protein dynamics during various biological processes. The nuclear localization of NCOA3 depends on the phosphorylation and two nuclear localization signals in its $\mathrm{N}$-terminal bHLH domain ${ }^{50}$. The stability of NCOA3 can affect the nuclear localization of NCOA3 through the proteasome-dependent pathway by recognizing two sites (K17 and R18) of the N-terminal bHLH domain of NCOA3 ${ }^{51}$. We found that the amount of nuclear NCOA3 binding to the TERT promoter in different four HCC cell lines is not consistent with the total protein levels in these cells (Fig. 1D). The possible reason is that the phosphorylation modification and stability of NCOA3 do not constant in these cells, thereby leading to a different binding amount in the TERT promoter.

Somatic mutation in TERT promoter frequently occurs in HCC patients (59\%), the most two hot mutation spots were -124 and $-146 \mathrm{bp}$ from the ATG start site $^{52}$. We found that NCOA3 bound to the -234 to $-144 \mathrm{bp}$ from the transcription site of the TERT promoter $(-291$ to $-201 \mathrm{bp}$ from the ATG start site). And, the hot spots -124 resides the sequence of SP1 binding. So, the mutation in the TERT promoter may have a function that facilitates the recruitment of transcription factors or cofactors into the TERT promoter to activate its expression. However, the potential mechanism and clinical significance of TERT promoter mutation which enhances NCOA3 binding and active TERT expression and cell growth in HCC cells need further exploration.

\section{Acknowledgements}

This work was supported by the funds from the National Natural Science Foundation of China (81872267, 81702761, and 81972569), and the Natural Science Foundation of Guangdong Province (2018A030313542).

\section{Author details}

${ }^{1}$ Sun Yat-sen University Cancer Center; State Key Laboratory of Oncology in South China; Collaborative Innovation Center of Cancer Medicine, Guangzhou, China. Institute of Neuroscience and Department of Neurology of the Second Affiliated Hospital of Guangzhou Medical University, Key Laboratory of

Neurogenetics and Channelopathies of Guangdong Province and the Ministry of Education of China, Guangzhou, China. ${ }^{3}$ The Third Affiliated Hospital, Sun 
Yat-Sen University, Guangzhou, China. ${ }^{4}$ Shunde Hospital of Southern Medical University, Foshan, Guangdong, China. ${ }^{5}$ Institute of Cancer Stem Cell, Dalian Medical University, Dalian, China

\section{Author contributions}

W.L., W.D., R.L., and L.L. conceived and designed the project. W.L., Y.Y., Z.Z., Q.Z., Q.L., S.S., M.L., M.C., Y.L., Y.H., W.D., R.L., and L.L. performed the experiments and analyzed and interpreted the data. W.L., W.D., R.L., and L.L. wrote and revised the paper. All authors read and approved the final paper.

\section{Conflict of interest}

The authors declare that they have no conflict of interest.

\section{Publisher's note}

Springer Nature remains neutral with regard to jurisdictional claims in published maps and institutional affiliations.

Supplementary Information accompanies this paper at (https://doi.org/ 10.1038/s41419-020-03218-x)

Received: 21 June 2020 Revised: 5 November 2020 Accepted: 9 November 2020

Published online: 25 November 2020

\section{References}

1. Lozano, R. et al. Global and regional mortality from 235 causes of death for 20 age groups in 1990 and 2010: a systematic analysis for the Global Burden of Disease Study 2010. Lancet 380, 2095-2128 (2012).

2. Marquardt, J. U. \& Thorgeirsson, S. S. SnapShot: hepatocellular carcinoma. Cancer Cell 25, $550 . e 551$ (2014).

3. Bosetti, C., Turati, F. \& La Vecchia, C. Hepatocellular carcinoma epidemiology. Best. Pract. Res Clin. Gastroenterol. 28, 753-770 (2014).

4. Kulik, L. M. \& Chokechanachaisakul, A. Evaluation and management of hepatocellular carcinoma. Clin. Liver Dis. 19, 23-43 (2015).

5. Facciorusso, A. et al. Mitochondrial signaling and hepatocellular carcinoma: molecular mechanisms and therapeutic implications. Curr. Pharm. Des. 22, 2689-2696 (2016).

6. Torrecilla, S. \& Llovet, J. M. New molecular therapies for hepatocellular carcinoma. Clin. Res. Hepatol. Gastroenterol. 39, S80-S85 (2015).

7. Hanahan, D. \& Weinberg, R. A. Hallmarks of cancer: the next generation. Cell 144, 646-674 (2011).

8. Low, K. C. \& Tergaonkar, V. Telomerase: central regulator of all of the hallmarks of cancer. Trends Biochem. Sci. 38, 426-434 (2013).

9. Choi, J. et al. TERT promotes epithelial proliferation through transcriptional control of a Myc- and Wnt-related developmental program. PLoS Genet 4, e10 (2008).

10. Kim, N. W. et al. Specific association of human telomerase activity with immortal cells and cancer. Science 266, 2011-2015 (1994).

11. Yin, L., Hubbard, A. K. \& Giardina, C. NF-kappa B regulates transcription of the mouse telomerase catalytic subunit. J. Biol. Chem. 275, 36671-36675 (2000).

12. Zhang, Y., Toh, L., Lau, P. \& Wang, X. Human telomerase reverse transcriptase (hTERT) is a novel target of the Wnt/beta-catenin pathway in human cancer. J. Biol. Chem. 287, 32494-32511 (2012).

13. Oh, B. K. et al. Quantitative assessment of hTERT mRNA expression in dysplastic nodules of HBV-related hepatocarcinogenesis. Am. J. Gastroenterol. 101 831-838 (2006).

14. Zhou, X. U., Lu, J. \& Zhu, H. Correlation between the expression of hTERT gene and the clinicopathological characteristics of hepatocellular carcinoma. Oncol. Lett. 11, 111-115 (2016).

15. Anzick, S. L. et al. AIB1, a steroid receptor coactivator amplified in breast and ovarian cancer. Science 277, 965-968 (1997).

16. Chen, H. et al. Nuclear receptor coactivator ACTR is a novel histone acetyltransferase and forms a multimeric activation complex with P/CAF and CBP/ p300. Cell 90, 569-580 (1997).

17. Cavailles, V., Dauvois, S., Danielian, P. S. \& Parker, M. G. Interaction of proteins with transcriptionally active estrogen receptors. Proc. Natl Acad. Sci. USA 91, 10009-10013 (1994)
18. Qin, L. et al. The AIB1 oncogene promotes breast cancer metastasis by activation of PEA3-mediated matrix metalloproteinase 2 (MMP2) and MMP9 expression. Mol. Cell Biol. 28, 5937-5950 (2008).

19. Louie, M. C., Zou, J. X., Rabinovich, A. \& Chen, H. W. ACTR/AIB1 functions as an E2F1 coactivator to promote breast cancer cell proliferation and antiestrogen resistance. Mol. Cell Biol. 24, 5157-5171 (2004).

20. Mussi, P., Yu, C., O'Malley, B. W. \& Xu, J. Stimulation of steroid receptor coactivator-3 (SRC-3) gene overexpression by a positive regulatory loop of E2F1 and SRC-3. Mol. Endocrinol. 20, 3105-3119 (2006).

21. Long, W. et al. ERK3 signals through SRC-3 coactivator to promote human lung cancer cell invasion. J. Clin. Invest. 122, 1869-1880 (2012).

22. Zhao, W. et al. Steroid receptor coactivator-3 regulates glucose metabolism in bladder cancer cells through coactivation of hypoxia inducible factor 1alpha. J. Biol. Chem. 289, 11219-11229 (2014).

23. Rajagopalan, D. et al. TIP60 represses telomerase expression by inhibiting Sp1 binding to the TERT promoter. PLoS Pathog. 13, e1006681 (2017).

24. Liao, Y. et al. Dysregulated Sp1/miR-130b-3p/HOXA5 axis contributes to tumor angiogenesis and progression of hepatocellular carcinoma. Theranostics 10, 5209-5224 (2020).

25. Kong, X. et al. ZBP-89 and Sp1 contribute to Bak expression in hepatocellular carcinoma cells. BMC Cancer 18, 419 (2018).

26. Zhang, X. et al. Sp1-regulated transcription of RasGRP1 promotes hepatocellular carcinoma (HCC) proliferation. Liver Int. 38, 2006-2017 (2018).

27. Torres-Arzayus, M. I. et al. High tumor incidence and activation of the PI3K/AKT pathway in transgenic mice define AlB1 as an oncogene. Cancer Cell 6, 263-274 (2004).

28. Zhou, H. J. et al. SRC-3 is required for prostate cancer cell proliferation and survival. Cancer Res. 65, 7976-7983 (2005).

29. Xu, J., Wu, R. C. \& O'Malley, B. W. Normal and cancer-related functions of the p160 steroid receptor co-activator (SRC) family. Nat. Rev. Cancer 9, 615-630 (2009).

30. $\mathrm{Xu}, \mathrm{Y}$. et al. Overexpression of transcriptional coactivator AlB1 promotes hepatocellular carcinoma progression by enhancing cell proliferation and invasiveness. Oncogene 29, 3386-3397 (2010).

31. Qin, G. et al. NPM1 upregulates the transcription of PD-L1 and suppresses T cell activity in triple-negative breast cancer. Nat. Commun. 11,1669,

32. Carey, M. F., Peterson, C. L. \& Smale, S. T. Chromatin immunoprecipitation (ChIP). Cold Spring Harb. Protoc. https://doi.org/10.1101/pdb.prot5279 (2009).

33. Verdun, R. E. \& Karlseder, J. Replication and protection of telomeres. Nature 447, 924-931 (2007).

34. Blackburn, E. H. The end of the (DNA) line. Nat. Struct. Biol. 7, 847-850 (2000).

35. Li, S. et al. Rapid inhibition of cancer cell growth induced by lentiviral delivery and expression of mutant-template telomerase RNA and anti-telomerase short-interfering RNA. Cancer Res. 64, 4833-4840 (2004).

36. Hrdlickova, R., Nehyba, J. \& Bose, H. R. Jr. Alternatively spliced telomerase reverse transcriptase variants lacking telomerase activity stimulate cell proliferation. Mol. Cell Biol. 32, 4283-4296 (2012).

37. Falchetti, M. L. et al. Inhibition of telomerase in the endothelial cells disrupts tumor angiogenesis in glioblastoma xenografts. Int. J. Cancer 122, 1236-1242 (2008).

38. Zhou, L., Zheng, D., Wang, M. \& Cong, Y. S. Telomerase reverse transcriptase activates the expression of vascular endothelial growth factor independent of telomerase activity. Biochem. Biophys. Res. Commun. 386, 739-743 (2009).

39. Bermudez, Y. et al. Telomerase confers resistance to caspase-mediated apoptosis. Clin. Inter. Aging 1, 155-167 (2006).

40. Singhapol, C. et al. Mitochondrial telomerase protects cancer cells from nuclear DNA damage and apoptosis. PLoS ONE 8, e52989 (2013).

41. Wang, Y. et al. Prognostic significance of c-myc and AIB1 amplification in hepatocellular carcinoma. A broad survey using high-throughput tissue microarray. Cancer 95, 2346-2352 (2002).

42. Takakura, M. et al. Cloning of human telomerase catalytic subunit (hTERT) gene promoter and identification of proximal core promoter sequences essential for transcriptional activation in immortalized and cancer cells. Cancer Res. 59, 551-557 (1999).

43. Tang, Z. et al. Molecular cloning and characterization of a human gene involved in transcriptional regulation of hTERT. Biochem. Biophys. Res. Commun. 324, 1324-1332 (2004).

44. Daniel, M., Peek, G. W. \& Tollefsbol, T. O. Regulation of the human catalytic subunit of telomerase (hTERT). Gene 498, 135-146 (2012). 
45. Kyo, S. et al. Sp1 cooperates with c-Myc to activate transcription of the human telomerase reverse transcriptase gene (hTERT). Nucleic Acids Res. 28, 669-677 (2000).

46. Park, N. H., Guo, W., Kim, H. R., Kang, M. K. \& Park, N. H. c-Myc and Sp1/3 are required for transactivation of hamster telomerase catalytic subunit gene promoter. Int. J. Oncol. 19, 755-761 (2001).

47. Zhao, Y., Cheng, D., Wang, S. \& Zhu, J. Dual roles of c-Myc in the regulation of hTERT gene. Nucleic Acids Res. 42, 10385-10398 (2014).

48. Liao, L. et al. Molecular structure and biological function of the canceramplified nuclear receptor coactivator SRC-3/AIB1. J. Steroid Biochem. Mol. Biol. 83, 3-14 (2002)
49. Bersten, D. C., Sullivan, A. E., Peet, D. J. \& Whitelaw, M. L. bHLH-PAS proteins in cancer. Nat. Rev. Cancer 13, 827-841 (2013).

50. Wu, R. C. et al. Selective phosphorylations of the SRC-3/AIB1 coactivator integrate genomic reponses to multiple cellular signaling pathways. Mol. Cell 15, 937-949 (2004).

51. Li, C. et al. Specific amino acid residues in the basic helix-loop-helix domain of SRC-3 are essential for its nuclear localization and proteasome-dependent turnover. Mol. Cell Biol. 27, 1296-1308 (2007).

52. Nault, J. C. et al. High frequency of telomerase reverse-transcriptase promoter somatic mutations in hepatocellular carcinoma and preneoplastic lesions. Nat. Commun. 4, 2218 (2013). 